\title{
Reflexivity of Spaces of Polynomials on Direct Sums of Banach Spaces
}

\author{
By \\ Adriano L. Aguiar* and Luiza A. Moraes **
}

\begin{abstract}
Let $P\left({ }^{n} E, F\right)$ be the space of the continuous $n$-homogeneous polynomials from $E$ into $F$ and $H_{b}(E, F)$ be the space of the holomorphic mappings from $E$ into $F$ that are bounded in the bounded subsets of $E$, both spaces endowed with the topology $\tau_{b}$ of uniform convergence on the bounded subsets of $E$. The reflexivity of $P\left({ }^{n} E, F\right)$ is studied in connection with the density of the space of the finite type $n$-homogeneous polynomials in $P\left({ }^{n} E, F\right)$ and in connection with the equality $\left[P\left({ }^{n} E, F\right), \tau_{b}\right]^{\prime}=\left[P\left({ }^{n} E, F\right), \tau_{0}\right]^{\prime}$ in case $E$ is a reflexive countable direct sum of complex Banach spaces and $F$ is a reflexive complex Banach space. The reflexivity of $H_{b}(E)$ is also considered.
\end{abstract}

\section{$\S 1$. Introduction}

If $E^{\prime}$ is the topological dual of a complex locally convex space $E$, we will write $E_{w}^{\prime}=\left(E^{\prime}, \sigma\left(E^{\prime}, E^{\prime \prime}\right)\right)$ where $\sigma\left(E^{\prime}, E^{\prime \prime}\right)$ denotes the weak topology on $E^{\prime}$ and $E_{b}^{\prime}=\left(E^{\prime}, \beta\left(E^{\prime}, E\right)\right)$ where $\beta\left(E^{\prime}, E\right)$ denotes the topology on $E^{\prime}$ of uniform convergence on the bounded subsets of $E$. Given complex locally convex spaces $E$ and $F$, let $P\left({ }^{n} E, F\right)$ be the space of continuous n-homogeneous polynomials from $E$ into $F$. As usual, if $E=\mathbb{C}$ we will write $P\left({ }^{n} E\right)$.

\footnotetext{
Communicated by H. Okamoto. Received December 17, 2007. Revised February 29, 2008, March 18, 2008.

2000 Mathematics Subject Classification(s): Primary: 46G25; Secondary: 46G20.

Key words: n-Homogeneous polynomial, reflexivity, direct sum.

*C.P. 1010, CEP 57022-970 Maceió, AL, Brazil.

Supported in part by CAPES, Brazil, Scholarship.

e-mail: adriano_aguiar@hotmail.com

**Instituto de Matemática, Universidade Federal do Rio de Janeiro, C. P. 68530, CEP 21945-970 Rio de Janeiro, RJ, Brazil.

Supported in part by CNPq, Brazil, Research Grant 309699/2006-1.

e-mail: luiza@im.ufrj.br
}

(c) 2009 Research Institute for Mathematical Sciences, Kyoto University. All rights reserved. 
We shall consider in $P\left({ }^{n} E, F\right)$ the standard topologies $\tau_{0}$ and $\tau_{b}$ where $\tau_{0}$ denotes the compact open topology and $\tau_{b}$ denotes the topology of uniform convergence on the bounded subsets of $E$. We refer to [16] for the definition of the topology $\tau_{\omega}$ on $P\left({ }^{n} E, F\right)$. In general we have $\tau_{0} \preceq \tau_{b} \preceq \tau_{\omega}$. The topologies $\tau_{b}$ and $\tau_{\omega}$ coincide in $P\left({ }^{n} E, F\right)$ whenever $E$ is a countable inductive limit of normed spaces (cf. Example 1.25, p. 25-26 in [16]).

The reflexivity of spaces of continuous scalar valued homogeneous polynomials on reflexive Banach spaces was first studied by R. Ryan in his thesis (see [26] and [3]). After his pioneering work, many authors considered the problem of finding necessary and sufficient condition for the space $P\left({ }^{n} E\right)$ to be reflexive (see [3], [4], [5], [18] and [13]). Alencar, Aron and Dineen showed in [5] that $P\left({ }^{n} T^{*}\right)$ is reflexive for every $n$, where $T^{*}$ is the Tsirelson's space (see [27]). This was the first example of an infinite dimensional Banach space $E$ for which the space $P\left({ }^{n} E\right)$ is reflexive for every $n$. Aron, Moraes and Ryan proved in [8] that if $E$ is a quotient of $T^{*}$ then $P\left({ }^{n} E\right)$ is reflexive. The vector valued case was first considered by Alencar who proved in [4] that if $E$ and $F$ are reflexive Banach spaces with the approximation property, then $P\left({ }^{n} E, F\right)$ is reflexive if and only if each $P \in P\left({ }^{n} E, F\right)$ is weakly continuous on bounded sets. Later Jaramillo and Moraes showed in [22] that the above necessary and sufficient conditions for the reflexivity of $P\left({ }^{n} E, F\right)$ remains valid when only $E$ is assumed to have the approximation property. The reflexivity of the space $H_{b}(E, F)$ of the holomorphic mappings from $E$ into $F$ that are bounded on the bounded sets was also considered in [22].

We define $P_{w u}\left({ }^{n} E, F\right)$ as the space of the elements of $P\left({ }^{n} E, F\right)$ that are uniformly weakly continuous on the bounded subsets of $E$ and $P_{f}\left({ }^{n} E, F\right)$ as the space of the finite type n-homogeneous polynomials i.e., $P_{f}\left({ }^{n} E, F\right)=$ $\operatorname{span}\left\{\varphi^{n} \otimes b, \varphi \in E^{\prime}, b \in F\right\} \quad$ where $\varphi^{n} \otimes b(z)=\varphi^{n}(z) \cdot b$. The closure of $P_{f}\left({ }^{n} E, F\right)$ in $\left[P\left({ }^{n} E, F\right), \tau_{b}\right]$ will be denoted by $\overline{P_{f}\left({ }^{n} E, F\right)}$. For locally convex spaces $E$ and $F$, Boyd showed that $\overline{P_{f}\left({ }^{n} E, F\right)}=P_{w u}\left({ }^{n} E, F\right)$ whenever $E^{\prime}$ has the approximation property (cf. [10], Proposition 7). The space of all $P \in P\left({ }^{n} E, F\right)$ which maps weakly convergent sequences in $E$ to convergent sequences in $F$ will be denoted by $P_{w s c}\left({ }^{n} E, F\right)$. It is clear that $P_{w u}\left({ }^{n} E, F\right) \subset$ $P_{w s c}\left({ }^{n} E, F\right)$.

In this paper we are going to study the density of $P_{f}\left({ }^{n} E, F\right)$ in $P\left({ }^{n} E, F\right)$ in connection with the reflexivity of $\left[P\left({ }^{n} E, F\right), \tau_{b}\right]$ and the reflexivity of $H_{b}(E, F)$ in the case $E$ is an countable direct sum of Banach spaces and $F$ is a Banach space.

We refer to [15], [16] and [23] for background information on polynomials 
and holomorphic mappings over locally convex spaces, in particular for the definitions of the topologies $\tau_{\delta}$ and $\tau_{\omega}$ in $H(E, F)$.

The authors want to thank Seán Dineen and Christopher Boyd for many helpful conversations concerning this paper. They want to acknowledge the referee for valuable suggestions that improved this paper.

\section{§2. Reflexivity of Spaces of Polynomials in Direct Sums of Banach Spaces}

Given any countable family of complex Banach spaces $E_{i}$, let $E=\sum_{i=1}^{\infty} E_{i}$ be the direct sum of the spaces $E_{i}$. For every $m \in \mathbb{N}$ let $F_{m}=\sum_{i=1}^{m} E_{i}$. We remark that $E$ is the strict inductive limit of the countable family of complex Banach spaces $F_{m}$ and consequently $E$ is a (DF)-space which is barrelled and bornological.

Remark 1. By using the polarization formula and Example 1.25 in [16] it is easy to verify that for each $n \in \mathbb{N}$ we have that $P \in P\left({ }^{n} E, F\right)$ if and only if $P \mid F_{m} \in P\left({ }^{n} F_{m}, F\right)$ for every $m \in \mathbb{N}$.

Proposition 2.1. Let $E=\sum_{i=1}^{\infty} E_{i}$ where $E_{i}$ is a complex Banach space for every $i \in \mathbb{N}$ and $F$ be a complex Banach space. Then, for each $n \in \mathbb{N}$, we have that $P_{w u}\left({ }^{n} E, F\right)=P\left({ }^{n} E, F\right)$ if and only if $P_{w u}\left({ }^{n} F_{m}, F\right)=$ $P\left({ }^{n} F_{m}, F\right)$ for all $m \in \mathbb{N}$.

Proof. The proof uses the above remark, the fact that $E$ is a regular inductive limit of the spaces $F_{m}$ and the Hahn-Banach Theorem.

The following lemma was proved by Dineen in the scalar case (see the proof of Proposition 3.1 in [14] or Proposition 4 in [17]). The same argument works to prove the vectorial case.

Lemma 2.1. Let $E=\sum_{i=1}^{\infty} E_{i}$ where $E_{i}$ is a complex Banach space for every $i \in \mathbb{N}$ and $F$ be a complex Banach space. Let $p$ be a $\tau_{\delta}$-continuous seminorm on $H(E, F)$. Then there exists a positive integer $m$ such that $f \in$ $H(E, F)$ and $f \mid F_{m}=0$ imply $p(f)=0$.

We remark that the topology $\tau_{\delta}$ in $H(E, F)$ induces the topology $\tau_{\omega}$ in $P\left({ }^{n} E, F\right)$ if $E$ is a locally convex space and $F$ is a Banach space (cf. Proposition 2.41 in [15]) and we recall that the topologies $\tau_{b}$ and $\tau_{\omega}$ coincide in $P\left({ }^{n} E, F\right)$ whenever $E=\sum_{i=1}^{\infty} E_{i}$ where $E_{i}$ is a complex Banach space for every $i \in \mathbb{N}$ and $F$ be a complex Banach space. 
Proposition 2.2. Let $E=\sum_{i=1}^{\infty} E_{i}$ where $E_{i}$ is a complex Banach space for every $i \in \mathbb{N}$ and $F$ be a complex Banach space. Then for each $n \in \mathbb{N}$ we have that $\left[P\left({ }^{n} E, F\right), \tau_{b}\right]^{\prime}=\left[P\left({ }^{n} E, F\right), \tau_{0}\right]^{\prime}$ if and only if $\left[P\left({ }^{n} F_{m}, F\right), \tau_{b}\right]^{\prime}=$ $\left[P\left({ }^{n} F_{m}, F\right), \tau_{0}\right]^{\prime}$, for all $m \in \mathbb{N}$.

Proof. Suppose that $\left[P\left({ }^{n} E, F\right), \tau_{b}\right]^{\prime}=\left[P\left({ }^{n} E, F\right), \tau_{0}\right]^{\prime}$. Fix $m \in \mathbb{N}$. From $\tau_{b} \succeq \tau_{0}$ it is clear that $\left[P\left({ }^{n} F_{m}, F\right), \tau_{0}\right]^{\prime} \subset\left[P\left({ }^{n} F_{m}, F\right), \tau_{b}\right]^{\prime}$. Now, given $\phi \in\left[P\left({ }^{n} F_{m}, F\right), \tau_{b}\right]^{\prime}$, we define $\psi: P\left({ }^{n} E, F\right) \longrightarrow \mathbb{C}$ by letting $\psi(P)=$ $\phi\left(P \mid F_{m}\right)$. The linearity of $\psi$ is clear and the $\tau_{b}$-continuity of $\psi$ follows from the $\tau_{b}$-continuity of $\phi$ and from the fact that $\tau_{b}$-convergence in $P\left({ }^{n} E, F\right)$ implies $\tau_{b}$-convergence in $P\left({ }^{n} F_{m}, F\right)$. Now we use the hypothesis to get $\psi \in$ $\left[P\left({ }^{n} E, F\right), \tau_{0}\right]^{\prime}$. Finally, we claim that $\phi \in\left[P\left({ }^{n} F_{m}, F\right), \tau_{0}\right]^{\prime}$. Indeed, given a net $\left(Q_{\lambda}\right)_{\lambda \in \Lambda}$ in $P\left({ }^{n} F_{m}, F\right)$ such that $Q_{\lambda} \rightarrow Q$ in $\left[P\left({ }^{n} F_{m}, F\right), \tau_{0}\right]$, let $\tilde{Q}_{\lambda}:=Q_{\lambda} \circ \pi_{m}$ and $\tilde{Q}:=Q \circ \pi_{m}$ where $\pi_{m}$ is the projection of $E$ onto $F_{m}$. It is clear that $\tilde{Q}_{\lambda} \rightarrow \tilde{Q}$ in $\left[P\left({ }^{n} E, F\right), \tau_{0}\right]$, since for all compact $K \subset E$ we have $\left\|\tilde{Q}_{\lambda}-\tilde{Q}\right\|_{K}=$ $\left\|Q_{\lambda}-Q\right\|_{\pi_{m}(K)}$ with $\pi_{m}(K)$ compact. Then $\psi\left(\tilde{Q}_{\lambda}\right) \rightarrow \psi(\tilde{Q})$ and consequently $\phi\left(Q_{\lambda}\right) \rightarrow \phi(Q)$. Conversely take $\psi \in\left[P\left({ }^{n} E, F\right), \tau_{b}\right]^{\prime}$. Since the topology induced in $P\left({ }^{n} E, F\right)$ by the topology $\tau_{\delta}$ in $H(E, F)$ coincides with $\tau_{b}$, by the Hahn-Banach Theorem there exists $\tilde{\psi} \in\left[H(E), \tau_{\delta}\right]^{\prime}$ such that $\tilde{\psi} \mid P\left({ }^{n} E, F\right)=\psi$. So $p(f)=|\tilde{\psi}(f)|$ is a $\tau_{\delta}$-continuous seminorm on $H(E, F)$ and therefore, by Lemma 2.1, there exists $m_{0}$ such that $p(f)=0$ whenever $f \in H(E, F)$ satisfies $f \mid F_{m_{0}}=0$.

Define $\mu:\left[P\left({ }^{n} F_{m_{0}}, F\right), \tau_{b}\right] \longrightarrow \mathbb{C}$ by letting $\mu(Q)=\psi(\tilde{Q})$ where $\tilde{Q}=$ $Q \circ \pi_{m_{0}}$. By hypothesis $\mu$ is $\tau_{b}$-continuous if and only if $\mu$ is $\tau_{0}$-continuous. Moreover if $Q_{\lambda} \rightarrow Q$ in $\left[P\left({ }^{n} F_{m_{0}}, F\right), \tau_{b}\right]$, then $\tilde{Q}_{\lambda} \rightarrow \tilde{Q}$ in $\left[P\left({ }^{n} E, F\right), \tau_{b}\right]$, as $\left\|\tilde{Q}_{\lambda}-\tilde{Q}\right\|_{B}=\left\|Q_{\lambda}-Q\right\|_{\pi_{m_{0}}(B)}$ and $\pi_{m_{0}}(B)$ is bounded in $F_{m_{0}}$ whenever $B$ is a bounded subset of $E$. Therefore $\mu\left(Q_{\lambda}\right) \rightarrow \mu(Q)$, and so $\mu$ is $\tau_{b^{-}}$ continuous and consequently $\tau_{0}$-continuous. Now if $P_{\alpha} \rightarrow P$ in $\left[P\left({ }^{n} E, F\right), \tau_{0}\right]$, then $P_{\alpha}\left|F_{m_{0}} \rightarrow P\right| F_{m_{0}}$ in $\left[P\left({ }^{n} F_{m_{0}}, F\right), \tau_{0}\right]$ and then $\mu\left(P_{\alpha} \mid F_{m_{0}}\right) \rightarrow \mu\left(P \mid F_{m_{0}}\right)$. But $\mu\left(P_{\alpha} \mid F_{m_{0}}\right)=\psi\left(\widetilde{P_{\alpha} \mid F_{m_{0}}}\right)=\psi\left(P_{\alpha}\right)$ and $\mu\left(P \mid F_{m_{0}}\right)=\psi\left(\widetilde{P \mid F_{m_{0}}}\right)=\psi(P)$, as $\left(\widetilde{P_{\alpha} \mid F_{m_{0}}}-P_{\alpha}\right) \mid F_{m_{0}}=0$ and $\left(\widetilde{P \mid F_{m_{0}}}-P\right) \mid F_{m_{0}}=0$. So, $\psi\left(P_{\alpha}\right) \rightarrow \psi(P)$ and we have $\left[P\left({ }^{n} E, F\right), \tau_{b}\right]^{\prime} \subset\left[P\left({ }^{n} E, F\right), \tau_{0}\right]^{\prime}$. Finally, $\tau_{b} \succeq \tau_{0}$ gives the converse inclusion.

Proposition 2.3. Let $E=\sum_{i=1}^{\infty} E_{i}$ where $E_{i}$ is a complex Banach space for every $i \in \mathbb{N}$ and $F$ be a complex Banach space. Then for each $n \in \mathbb{N}, \quad\left[P\left({ }^{n} E, F\right), \tau_{b}\right]$ is quasi complete for its weak topology if and only if $\left[P\left({ }^{n} F_{m}, F\right), \tau_{b}\right]$ is quasi complete for its weak topology for all $m \in \mathbb{N}$.

Proof. Suppose that $\left[P\left({ }^{n} F_{m}, F\right), \tau_{b}\right]$ is quasi complete for its weak topo- 
logy for every $m \in \mathbb{N}$. Let $\left(P_{\alpha}\right)_{\alpha} \subset P\left({ }^{n} E, F\right)$ be a bounded Cauchy net for the weak topology of $\left[P\left({ }^{n} E, F\right), \tau_{b}\right]$. We claim that $\left(P_{\alpha} \mid F_{m}\right)_{\alpha}$ is a bounded Cauchy net for the weak topology of $\left[P\left({ }^{n} F_{m}, F\right), \tau_{b}\right]$, for all $m \in \mathbb{N}$. Indeed, $\left(P_{\alpha} \mid F_{m}\right)_{\alpha}$ is bounded since the unit ball of $F_{m}$ is a bounded subset of $E$ and $\left(P_{\alpha}\right)_{\alpha}$ is a bounded net in $\left[P\left({ }^{n} E, F\right), \tau_{b}\right]$. As in the last proposition, given $\phi \in\left[P\left({ }^{n} F_{m}, F\right), \tau_{b}\right]^{\prime}$, the mapping $\psi:\left[P\left({ }^{n} E, F\right), \tau_{b}\right] \longrightarrow \mathbb{C}$ defined by $\psi(Q)=$ $\phi\left(Q \mid F_{m}\right)$ belongs to $\left[P\left({ }^{n} E, F\right), \tau_{b}\right]^{\prime}$. Therefore $\left(\psi\left(P_{\alpha}\right)\right)_{\alpha}=\left(\phi\left(P_{\alpha} \mid F_{m}\right)\right)_{\alpha}$ is a Cauchy net in $\mathbb{C}$. By hypothesis, for each $m$ there exists $P_{m} \in P\left({ }^{n} F_{m}, F\right)$ such that $P_{\alpha} \mid F_{m} \rightarrow P_{m}$ in the weak topology of $\left[P\left({ }^{n} F_{m}, F\right), \tau_{b}\right]$. In particular, $P_{\alpha} \mid F_{m}(x) \rightarrow P_{m}(x)$, for all $x \in F_{m}$. Let $P: E \longrightarrow F$ given by $P(x)=P_{m}(x)$ where $m$ is such that $x \in F_{m}$. It is clear that $P$ is well defined and $P \mid F_{m}=$ $P_{m}$, for all $m \in \mathbb{N}$. Moreover $P \in P\left({ }^{n} E, F\right)$ by Remark 1 above. Finally we are going to show that $P_{\alpha} \rightarrow P$ in the weak topology of $\left[P\left({ }^{n} E, F\right), \tau_{b}\right]$. Let $\psi \in\left[P\left({ }^{n} E, F\right), \tau_{b}\right]^{\prime}$. We saw already that there exists $m \in \mathbb{N}$ such that the mapping $\mu:\left[P\left({ }^{n} F_{m}, F\right), \tau_{b}\right] \longrightarrow \mathbb{C}$ defined by $\mu(Q)=\psi(\tilde{Q})$, where $\tilde{Q}=Q \circ \pi_{m}$, is continuous. Therefore $\mu\left(P_{\alpha} \mid F_{m}\right) \rightarrow \mu\left(P_{m}\right)$. We also saw that $\mu\left(P_{\alpha} \mid F_{m}\right)=$ $\psi\left(P_{\alpha}\right)$ and $\mu\left(P_{m}\right)=\psi(P)$. Consequently $\psi\left(P_{\alpha}\right) \rightarrow \psi(P)$, and this completes the proof.

Conversely assume that $\left[P\left({ }^{n} E, F\right), \tau_{b}\right]$ is quasi complete for its weak topology. Fix any $m \in \mathbb{N}$. Suppose that $\left(P_{\alpha}\right)_{\alpha}$ is a bounded Cauchy net for the weak topology of $\left[P\left({ }^{n} F_{m}, F\right), \tau_{b}\right]$. Let $\tilde{P}_{\alpha}=P_{\alpha} \circ \pi_{m}$. We show as in last proposition that for every $\psi \in\left[P\left({ }^{n} E, F\right), \tau_{b}\right]^{\prime}$ the mapping $\mu:\left[P\left({ }^{n} F_{m}, F\right), \tau_{b}\right] \longrightarrow \mathbb{C}$ defined by $\mu(Q)=\psi(\tilde{Q})$ is continuous. Therefore $\left(\mu\left(P_{\alpha}\right)\right)_{\alpha}$ is a Cauchy net in $\mathbb{C}$, and this means that $\left(\psi\left(\tilde{P}_{\alpha}\right)\right)_{\alpha}$ is a Cauchy net in $\mathbb{C}$. By hypothesis there exists $\tilde{P} \in P\left({ }^{n} E, F\right)$ such that $\tilde{P}_{\alpha} \rightarrow \tilde{P}$ in the weak topology of $\left[P\left({ }^{n} E, F\right), \tau_{b}\right]$. Finally, given $\phi \in\left[P\left({ }^{n} F_{m}, F\right), \tau_{b}\right]^{\prime}$, the mapping $\psi: P\left({ }^{n} E, F\right) \longrightarrow \mathbb{C}$ defined by $\psi(Q)=\phi\left(Q \mid F_{m}\right)$ is linear and $\tau_{b}$-continuous. Therefore, $\psi\left(\tilde{P}_{\alpha}\right) \rightarrow \psi(\tilde{P})$ and so $\phi\left(\tilde{P}_{\alpha} \mid F_{m}\right) \rightarrow \phi\left(\tilde{P} \mid F_{m}\right)$. The proof follows since $\tilde{P}_{\alpha} \mid F_{m}=P_{\alpha}$ and $\tilde{P} \mid F_{m} \in$ $P\left({ }^{n} F_{m}, F\right)$.

Theorem 2.1. Let $E=\sum_{i=1}^{\infty} E_{i}$ where $E_{i}$ is a reflexive complex Banach space for every $i \in \mathbb{N}$. Then for every reflexive complex Banach space $F$ and for each $n \in \mathbb{N}$ we have that $\left[P\left({ }^{n} E, F\right), \tau_{0}\right]^{\prime}=\left[P\left({ }^{n} E, F\right), \tau_{b}\right]^{\prime}$ if and only if $\left[P\left({ }^{n} E, F\right), \tau_{b}\right]$ is reflexive.

Proof. Suppose $\left[P\left({ }^{n} E, F\right), \tau_{0}\right]^{\prime}=\left[P\left({ }^{n} E, F\right), \tau_{b}\right]^{\prime}$. Then by Proposition 2.1 above and Theorem 3.1 of [24] we have that $\left[P\left({ }^{n} F_{m}, F\right), \tau_{b}\right]$ is reflexive for all $m \in \mathbb{N}$. So, by Proposition 3 in [21], p. $228,\left[P\left({ }^{n} F_{m}, F\right), \tau_{b}\right]$ is quasi complete for its weak topology for all $m \in \mathbb{N}$ and, by Proposition 2.3 above, $\left[P\left({ }^{n} E, F\right), \tau_{b}\right]$ 
is quasi complete for its weak topology. Now, using again Proposition 3 in [21], p. 228, and the fact that $\left[P\left({ }^{n} E, F\right), \tau_{b}\right]$ is a barrelled space whenever $E$ is a (DF)-space we get the reflexivity of $\left[P\left({ }^{n} E, F\right), \tau_{b}\right]$.

Conversely if $\left[P\left({ }^{n} E, F\right), \tau_{b}\right]$ is reflexive, we have that $\left[P\left({ }^{n} E, F\right), \tau_{b}\right]$ is quasi complete for its weak topology and, by Proposition $2.3,\left[P\left({ }^{n} F_{m}, F\right), \tau_{b}\right]$ is quasi complete for its weak topology for all $m \in \mathbb{N}$. So, $\left[P\left({ }^{n} F_{m}, F\right), \tau_{b}\right]$ is reflexive (recall that it is a barrelled space) and, by Theorem 3.1 of [24] we have that $\left[P\left({ }^{n} F_{m}, F\right), \tau_{0}\right]^{\prime}=\left[P\left({ }^{n} F_{m}, F\right), \tau_{b}\right]^{\prime}$ for all $m \in \mathbb{N}$. By Proposition 2.2 this means that $\left[P\left({ }^{n} E, F\right), \tau_{0}\right]^{\prime}=\left[P\left({ }^{n} E, F\right), \tau_{b}\right]^{\prime}$.

Remark 2. From the fact that $P\left({ }^{n} E, F\right)=P\left({ }^{n} E, F_{w}\right)$ when $E$ and $F$ are Banach spaces, it is easy to show that this equality remains true for $E=$ $\sum_{i=1}^{\infty} E_{i}\left(E_{i}\right.$ is a complex Banach space for every $\left.i \in \mathbb{N}\right)$ and $F$ a complex Banach space. If, in addition, $F$ is reflexive, following ideas of C. Boyd in [11] we can introduce in $P\left({ }^{n} E, F\right)$ a topology $\tau_{\gamma}$ by $\left[P\left({ }^{n} E, F\right), \tau_{\gamma}\right]=\left[P\left({ }^{n} E, F_{\gamma}\right), \tau_{0}\right]$ where $F_{\gamma}$ is the space $F$ endowed with the topology of uniform convergence on compact subsets of $F^{\prime}$. A net $\left(\tilde{Q}_{\lambda}\right)$ converges to $\tilde{Q}$ in $\left[P\left({ }^{n} E, F\right), \tau_{\gamma}\right]$ if $\sup _{x \in K} p \circ\left(\tilde{Q_{\lambda}}-\tilde{Q}\right)(x) \rightarrow 0$ for every compact subset $K$ of $E$ and for every continuous seminorm $p$ on $F_{\gamma}$ and it is known that $\tau_{\gamma}$ is weaker then $\tau_{0}$. Now the proof of Proposition 2.2 can be easily adapted to prove that for $E=\sum_{i=1}^{\infty} E_{i}$ (where $E_{i}$ is a complex Banach space for every $i \in \mathbb{N}$ ), $F$ a reflexive complex Banach space and for each $n \in \mathbb{N}$ we have that $\left[P\left({ }^{n} E, F\right), \tau_{b}\right]^{\prime}=\left[P\left({ }^{n} E, F\right), \tau_{\gamma}\right]^{\prime}$ if and only if $\left[P\left({ }^{n} F_{m}, F\right), \tau_{b}\right]^{\prime}=\left[P\left({ }^{n} F_{m}, F\right), \tau_{\gamma}\right]^{\prime}$, for all $m \in \mathbb{N}$.

Theorem 2.2. Let $E=\sum_{i=1}^{\infty} E_{i}$ where $E_{i}$ is a reflexive complex Banach space for every $i \in \mathbb{N}$. Then for every reflexive complex Banach space $F$ and for each $n \in \mathbb{N}$ we have that $\left[P\left({ }^{n} E, F\right), \tau_{\gamma}\right]^{\prime}=\left[P\left({ }^{n} E, F\right), \tau_{b}\right]^{\prime}$ if and only if $\left[P\left({ }^{n} E, F\right), \tau_{b}\right]$ is reflexive.

Proof. By the remarks following Theorem 8 of [11] we obtain that $\left[P\left({ }^{n} F_{m}, F\right), \tau_{b}\right]$ is reflexive if and only if $\left[P\left({ }^{n} F_{m}, F\right), \tau_{\gamma}\right]^{\prime}=\left[P\left({ }^{n} F_{m}, F\right), \tau_{b}\right]^{\prime}$. (Theorem 8 of [11] is actually true for holomorphic functions but, as the author remarks, the analogous result is also true for spaces of homogeneous polynomials.) By using this equivalence and Remark 2 we get that $\left[P\left({ }^{n} E, F\right), \tau_{\gamma}\right]^{\prime}=$ $\left[P\left({ }^{n} E, F\right), \tau_{b}\right]^{\prime}$ if and only if $\left[P\left({ }^{n} F_{m}, F\right), \tau_{b}\right]$ is reflexive for every $m \in \mathbf{N}$. Finally our theorem follows by using Theorem 3.1 of [24], Proposition 2.2 and Theorem 2.1.

Theorem 2.3. Let $E=\sum_{i=1}^{\infty} E_{i}$ where $E_{i}$ is a reflexive complex Banach space with the approximation property for every $i \in \mathbb{N}$ and let $F$ be a 
reflexive complex Banach space. For every $n \in \mathbb{N}$, the following conditions are equivalent:

(1) $\left[P\left({ }^{n} E, F\right), \tau_{0}\right]^{\prime}=\left[P\left({ }^{n} E, F\right), \tau_{b}\right]^{\prime}$.

(2) $\left[P\left({ }^{n} E, F\right), \tau_{b}\right]$ is reflexive.

(3) $\left[P\left({ }^{n} E, F\right), \tau_{\gamma}\right]^{\prime}=\left[P\left({ }^{n} E, F\right), \tau_{b}\right]^{\prime}$.

(4) $P_{w u}\left({ }^{n} E, F\right)=P\left({ }^{n} E, F\right)$.

(5) $\overline{P_{f}\left({ }^{n} E, F\right)}=P\left({ }^{n} E, F\right)$.

Proof. The equivalence between (1) and (2) and between (2) and (3) are established by Theorem 2.1 and Theorem 2.2, respectively. The equivalence between (1) and (5) follows by Theorem 3.1 of [24], Proposition 7 of [10], Proposition 2.2 and Proposition 2.1. The equivalence between (1) and (4) follows by Theorem 3.1 of [24], Proposition 2.2 and Proposition 2.1.

Remark 3. We remark that the implications $(5) \Rightarrow(4) \Rightarrow(3) \Leftrightarrow(2) \Leftrightarrow$ (1) are true even if the spaces $E_{i}$ don't have the approximation property and the implication $(2) \Rightarrow(4)$ remains true if the spaces $E_{i}$ have the compact approximation property instead of the approximation property.

We also remark that $E=\sum_{i=1}^{\infty} E_{i}$ has the approximation property if and only if $E_{i}$ has the approximation property for every $i \in \mathbb{N}$.

Proposition 2.4. Let $E=\mathbb{C}^{(\mathbb{N})}$ and let $F$ be an arbitrary complex Banach space. Then $\overline{P_{f}\left({ }^{n} E, F\right)}=P\left({ }^{n} E, F\right)$ for every $n \in \mathbb{N}$.

Proof. By Lemma 1 of [9] every bounded subset of $\mathbb{C}^{(\mathbb{N})}$ is finite dimensional and consequently every homogeneous polynomial $P: \mathbb{C}^{(\mathbb{N})} \rightarrow F$ is weakly continuous on bounded sets. Moreover, as the strong dual of $\mathbb{C}^{(\mathbb{N})}$ has the approximation property, by Proposition 7 of [10] we have $\overline{P_{f}\left({ }^{n} \mathbb{C}^{(\mathbb{N})}, F\right)}=$ $P_{w u}\left({ }^{n} \mathbb{C}^{(\mathbb{N})}, F\right)$ and the statement follows .

Proposition 2.5. Let $E=\sum_{i=1}^{\infty} E_{i}$ where $E_{i}$ is a complex Banach space for every $i \in \mathbb{N}$ and let $F$ be an arbitrary complex Banach space. If each $F_{m}$ does not contain a copy of $\ell_{1}$, then $P_{w s c}\left({ }^{n} E, F\right)=P_{w u}\left({ }^{n} E, F\right)$ for all $n \in \mathbb{N}$

Proof. If $\left(x_{n}\right)_{n}$ is a sequence in $E$ such that $x_{n} \rightarrow x$ as $n \rightarrow \infty$ in the topology $\sigma\left(E, E^{\prime}\right)$, then $B=\left\{x_{n}: n \in \mathbb{N}\right\} \cup\{x\}$ is a weakly bounded subset of $E$ and consequently it is bounded. It follows that $P_{w u}\left({ }^{n} E, F\right) \subset P_{w s c}\left({ }^{n} E, F\right)$. Conversely, take $P \in P_{w s c}\left({ }^{n} E, F\right)$. For each $m \in \mathbb{N}$ we have that if $\left(x_{n}\right)$ is an arbitrary sequence in $F_{m}$ which converges to $x \in F_{m}$ in the weak topology 
of $F_{m}$ then $\left(x_{n}\right)$ converges to $x$ in the weak topology of $E$. Consequently $P \mid F_{m} \in P_{w s c}\left({ }^{n} F_{m}, F\right)$ for every $m \in \mathbb{N}$. Moreover, as $F_{m}$ is a Banach space that does not contain a copy of $\ell_{1}$, by Proposition 2.12 of [7] we have that $P_{w s c}\left({ }^{n} F_{m}, F\right)=P_{w u}\left({ }^{n} F_{m}, F\right)$. So, $P \mid F_{m} \in P_{w u}\left({ }^{n} F_{m}, F\right)$ for all $m \in \mathbb{N}$ and by using the Hahn-Banach Theorem and the fact that $E$ is a regular inductive limit we get that $P \in P_{w u}\left({ }^{n} E, F\right)$.

We remark that the above result remains true for more general cases of regular inductive limits. For instance, it is true in case $E$ is a weakly compact regular inductive limit of Banach spaces $\left(E_{\alpha}\right)_{\alpha \in A}$ such that $\ell_{1} \nsubseteq E_{\alpha}$ for every $\alpha \in A$ and in case $E=\operatorname{ind}_{n \rightarrow \infty} E_{n}$ is a regular countable inductive limit with the property $M_{0}$ of Banach spaces $E_{n}$ such that $\ell_{1} \nsubseteq E_{n}$ for every $n \in \mathbb{N}$ (see Theorems 2.5 and 2.6 in [1]).

Aron and Dineen showed in [6] that the Tsirelson-James space $T_{J}^{*}$ satisfies the equation $\overline{P_{f}\left({ }^{n} T_{J}^{*}\right)}=P\left({ }^{n} T_{J}^{*}\right)$ for every $n \in \mathbb{N}$. For vector valued polynomials it is known that $\overline{P_{f}\left({ }^{n} T_{J}^{*}, F\right)}=P\left({ }^{n} T_{J}^{*}, F\right)$ for all $n \in \mathbb{N}$ whenever the Banach space $F$ has positive rank (see Example 2.3 in [20]). We recall that a Banach space has positive rank if there exists $\alpha \in(0,1)$ such that every sequence $\left(x_{n}\right)$ satisfying $\left\|\sum_{n \in B} x_{n}\right\| \leq c|B|^{\alpha}$ for $c \geq 0$ and for all finite $B \subset \mathbb{N}$ (where $|B|$ is the number of elements of $B$ ) converges with respect to the norm.

Proposition 2.6. Let $E=\sum_{i=1}^{\infty} E_{i}$ where $E_{i}=T_{J}^{*}$ for every $i \in \mathbb{N}$. Then $\overline{P_{f}\left({ }^{n} E, F\right)}=P\left({ }^{n} E, F\right)$ for all $n \in \mathbb{N}$ whenever $F$ is a complex Banach space which is Schur.

Proof. It is known (see Example 3 of [12] or the proof of Proposition 5.5 in [28], p.87) that $\overline{P_{f}\left({ }^{n} E\right)}=P\left({ }^{n} E\right)$, for all $n \in \mathbb{N}$. By Proposition 2.1, for every $n \in \mathbb{N}$, we have that $P_{w u}\left({ }^{n} F_{m}, F\right)=P\left({ }^{n} F_{m}, F\right)$ for all $m \in \mathbb{N}$. Consequently each $F_{m}$ does not contain a copy of $\ell_{1}$ and by Proposition 2.5 we get $P_{w s c}\left({ }^{n} E, F\right)=P_{w u}\left({ }^{n} E, F\right)$ for all $n \in \mathbb{N}$ and for all complex Banach space $F$. Now, all we have to show is $P_{w s c}\left({ }^{n} E, F\right)=P\left({ }^{n} E, F\right)$ whenever $F$ is a complex Banach space which is Schur. Indeed, given any $P \in P\left({ }^{n} E, F\right)$, for each $\varphi \in F^{\prime}$ we have that $\varphi \circ P \in P\left({ }^{n} E\right)=P_{w s c}\left({ }^{n} E\right)$. So, if $\left(x_{n}\right)$ converges weakly to $x$ in $E,\left(\varphi \circ P\left(x_{n}\right)\right)$ converges to $\varphi \circ P(x)$ in $\mathbb{C}$ and so the sequence $\left(P\left(x_{n}\right)\right)$ converges weakly to $P(x)$ in $F$. This completes the proof since $F$ is Schur.

Remark 4. We recall that the Tsirelson-James space is a quasi-reflexive Banach space that is not reflexive and so if $E=\sum_{i=1}^{\infty} E_{i}$ where $E_{i}=T_{J}^{*}$ for every $i \in \mathbb{N}$ then $E$ is not a reflexive space. Since $\overline{P_{f}\left({ }^{n} T_{J}^{*}\right)}=P\left({ }^{n} T_{J}^{*}\right)$ for all 
$n \in \mathbb{N}$, this space $E$ provides an example showing that the reflexivity of $E$ is necessary in Theorem 2.3. For $E=\sum_{i=1}^{\infty} T_{J}^{*}, \quad\left[P\left({ }^{1} E\right), \tau_{0}\right]^{\prime}=\left[E^{\prime}, \tau_{0}\right]^{\prime} \cong E \neq$ $E^{\prime \prime} \cong\left(E_{b}^{\prime}\right)^{\prime}=\left[P\left({ }^{1} E\right), \tau_{b}\right]^{\prime}$, as $T_{J}^{*}$ is not reflexive.

The same comment applies to $E=\sum_{i=1}^{\infty} c_{0}$.

Let $H_{b}(E, F)$ be the space of holomorphic mappings from $E$ into $F$ that are bounded on the bounded subsets of $E$ and let $H_{w u}(E, F)$ be the space of the holomorphic mappings from $E$ into $F$ that are uniformly weakly continuous on the bounded subsets of $E$, both endowed with the topology $\tau_{b}$ of uniform convergence on the bounded subsets of $E$.

Theorem 2.4. Let $E=\sum_{i=1}^{\infty} E_{i}$ where $E_{i}$ is a reflexive complex Banach space with the approximation property for every $i \in \mathbb{N}$ and let $F$ be a reflexive complex Banach space. The following conditions are equivalent:

(1) $\left[H_{b}(E, F), \tau_{b}\right]$ is reflexive.

(2) $H_{b}(E, F)=H_{w u}(E, F)$.

(3) $P_{w u}\left({ }^{n} E, F\right)=P\left({ }^{n} E, F\right)$ for all $n \in \mathbb{N}$.

(4) $\left[P\left({ }^{n} E, F\right), \tau_{b}\right]$ is reflexive for all $n \in \mathbb{N}$.

Proof. The reflexivity of $\left[H_{b}(E, F), \tau_{b}\right]$ implies the reflexivity of $\left[P\left({ }^{n} E, F\right), \tau_{b}\right]$ for every $n \in \mathbb{N}$ since $\left[P\left({ }^{n} E, F\right), \tau_{b}\right]$ is a closed subspace of $\left[H_{b}(E, F), \tau_{b}\right]$ and, by Theorem 4 in $[19],\left[H_{b}(E, F), \tau_{b}\right]$ is a Fréchet space. Conversely, by Propositions 3.1 and 2.3 in Chapter 4 of [28] we have that the reflexivity of $\left[P\left({ }^{n} E, F\right), \tau_{b}\right]$ for every $n \in \mathbb{N}$ implies the reflexivity of $\left[H_{b}(E, F), \tau_{b}\right]$. The equivalence between (2) and (3) follows, for instance, as a particular case of Proposition 2.1 in [2]. Finally, (3) and (4) are equivalent by Theorem 2.3.

We recall that given a Fréchet algebra $\mathcal{A}$, a multiplicative linear function $T: \mathcal{A} \rightarrow \mathbb{C}$ is called an homomorphism. For every $x \in E$, we will denote by $\delta_{x}$ the homomorphism $f \in H_{b}(E) \rightarrow f(x) \in \mathbb{C}$. We say that $\delta_{x}(x \in E)$ is an evaluation.

It is well known that $\left[H_{b}(E), \tau_{b}\right]$ is a Fréchet algebra. As a particular case of Theorem 3.9 in [2] we have the following:

Theorem 2.5. Let $E=\sum_{i=1}^{\infty} E_{i}$ where $E_{i}$ is a complex Banach space with the approximation property for every $i \in \mathbb{N}$. If $E$ is reflexive then each $\tau_{b}$-continuous homomorphism $T: H_{b}(E) \rightarrow \mathbb{C}$ is an evaluation, if and only if $P\left({ }^{n} E\right)=P_{w u}\left({ }^{n} E\right)$ for every $n \in \mathbb{N}$.

The next theorem provides a converse for this result. 
Theorem 2.6. Let $E=\sum_{i=1}^{\infty} E_{i}$ where $E_{i}$ is a complex Banach space with the approximation property for every $i \in \mathbb{N}$. If each $\tau_{b}$-continuous complex valued homomorphism on $H_{b}(E)$ is an evaluation, then $E$ is reflexive and $P\left({ }^{n} E\right)=P_{w u}\left({ }^{n} E\right)$ for every $n \in \mathbb{N}$.

Proof. If $E$ is not reflexive, then $F_{m}=\sum_{i=1}^{m} E_{i}$ is not reflexive for some $m \in \mathbb{N}$ and, by Theorem 1.2 in [25], there exists an homomorphism $T: H_{b}\left(F_{m}\right) \longrightarrow \mathbb{C}$ such that $T \neq \delta_{x}$ for any $x \in F_{m}$. Define $\bar{T}: H_{b}(E) \longrightarrow \mathbb{C}$ by $\bar{T}(g)=T\left(g \mid F_{m}\right)$ for every $g \in H_{b}(E)$. It is easy to see that $\bar{T}$ is a $\tau_{b^{-}}$ continuous homomorphism on $H_{b}(E)$ and by hypothesis we have $\bar{T}=\delta_{z}$ for some $z \in E$. Now, to each $f \in H_{b}\left(F_{m}\right)$ we can associate $\bar{f}=f \circ \pi_{m} \in H_{b}(E)$ and so we get

$$
T(f)=T\left(\bar{f} \mid F_{m}\right)=\bar{T}(\bar{f})=\delta_{z}(\bar{f})=\bar{f}(z)=f\left(\pi_{m}(z)\right)=\delta_{\pi_{m}(z)}(f)
$$

and this gives a contradiction.

So if every continuous complex valued homomorphism on $H_{b}(E)$ is an evaluation we have that $E$ is reflexive and by [2] Theorem 3.9 we have $P\left({ }^{n} E\right)=$ $P_{w u}\left({ }^{n} E\right)$ for every $n \in \mathbb{N}$.

Now, the next result is a immediate consequence of Theorems $2.4,2.5$ and 2.6 .

Theorem 2.7. Let $E=\sum_{i=1}^{\infty} E_{i}$ where $E_{i}$ is a complex Banach space with the approximation property for every $i \in \mathbb{N}$. Then every $\tau_{b}$-continuous homomorphism $T: H_{b}(E) \longrightarrow \mathbb{C}$ is an evaluation if and only if $H_{b}(E)$ is reflexive.

\section{References}

[1] A. L. Aguiar, Polinômios e Funções Holomorfas em Espaços Localmente Convexos, PhD Thesis, Universidade Federal do Rio de Janeiro, Rio de Janeiro, Brazil, 2003.

[2] A. L. Aguiar and L. A. Moraes, Algebras of holomorphic mappings in (DF)-spaces, Indag. Math. (N.S.) 18 (2007), no. 2, 161-175.

[3] R. Alencar, On reflexivity and basis for $P\left({ }^{n} X\right)$, Proc. Roy. Irish Acad. Sect. A 85 (1985), no. $2,131-138$.

[4] spaces (Mérida, 1992), 1-8, Contemp. Math., 144, Amer. Math. Soc., Providence, RI, 1993.

[5] R. Alencar, R. M. Aron and S. Dineen, A reflexive space of holomorphic functions in infinitely many variables, Proc. Amer. Math. Soc. 90 (1984), no. 3, 407-411.

[6] R. M. Aron and S. Dineen, Q-reflexive Banach spaces, Rocky Mountain J. Math. 27 (1997), no. 4, 1009-1025.

[7] R. M. Aron, C. Hervés and M. Valdivia, Weakly continuous mappings on Banach spaces, J. Funct. Anal. 52 (1983), no. 2, 189-204. 
[8] R. M. Aron, L. A. Moraes and R. A. Ryan, Factorization of holomorphic mappings in infinite dimensions, Math. Ann. 277 (1987), no. 4, 617-628.

[9] C. Boyd, Holomorphic mappings on $\mathbb{C}^{(I)}, I$ uncountable, Results Math. 36 (1999), no. $1-2,21-25$.

[10] , Duality and reflexivity of spaces of approximable polynomials on locally convex spaces, Monatsh. Math. 130 (2000), no. 3, 177-188.

[11] Preduals of spaces of vector-valued holomorphic functions, Czechoslovak Math. J. 53(128) (2003), no. 2, 365-376.

[12] C. Boyd, S. Dineen and M. Venkova, $Q$-reflexive locally convex spaces, Publ. Res. Inst. Math. Sci. 40 (2004), no. 1, 7-27.

[13] A. M. Davie and T. W. Gamelin, A theorem on polynomial-star approximation, Proc. Amer. Math. Soc. 106 (1989), no. 2, 351-356.

[14] S. Dineen, Holomorphic functions on locally convex topological vector spaces. I. Locally convex topologies on $\mathcal{H}(U)$, Ann. Inst. Fourier (Grenoble) 23 (1973), no. 1, 19-54.

[15] _ Complex analysis in locally convex spaces, North Holland Math. Studies 57, North-Holland, Amsterdam, 1981.

[16] _ Complex analysis on infinite-dimensional spaces, Springer Monogr. Math., Springer, London, 1999.

[17] S. Dineen and L. A. Moraes, Holomorphic functions on strict inductive limits of Banach spaces, Rev. Mat. Univ. Complut. Madrid 5 (1992), no. 2-3, 177-183.

[18] J. D. Farmer, Polynomial reflexivity in Banach spaces, Israel J. Math. 87 (1994), no. 1-3, $257-273$.

[19] P. Galindo, D. Garcia and M. Maestre, Holomorphic mappings of bounded type on (DF)spaces, in Progress in functional analysis (Peñiscola, 1990), 135-148, North-Holland, Amsterdam, 1992.

[20] D. Garcia, M. L. Lourenço, L. A. Moraes and O. W. Paques, The spectra of some algebras of analytic mappings, Indag. Math. (N.S.) 10 (1999), no. 3, 393-406.

[21] J. Horváth, Topological vector spaces and distributions. Vol. I, Addison-Wesley Publishing Co., Reading, Mass., 1966.

[22] J. A. Jaramillo and L. A. Moraes, Duality and reflexivity in spaces of polynomials, Arch. Math. (Basel) 74 (2000), no. 4, 282-293.

[23] J. Mujica, Complex analysis in Banach spaces, North-Holland Math. Stud., 120, NorthHolland, Amsterdam, 1986.

[24] Reflexive spaces of homogeneous polynomials, Bull. Polish Acad. Sci. Math. 49 (2001), no. 3, 211-222.

[25] Ideals of holomorphic functions on Tsirelson's space, Arch. Math. (Basel) 76 (2001), no. 4, 292-298.

[26] R. Ryan, Applications of Topological Tensor Product to Infinite Dimensional Holomorphy, Ph.D. Thesis, Trinity College Dublin, Dublin, Ireland, 1980.

[27] B. Tsirelson, Not every Banach space contains an imbedding of $l_{p}$ or $c_{0}$, Funct. Anal. Appl. 9 (1974) 138-141.

[28] M. Venkova, Q-reflexive Locally Convex Spaces, Ph.D. Thesis, University College Dublin, Dublin, Ireland, 2002. 